\title{
RÉFLEXION DISCURSIVE SUR LES INTERSTICES. DE L'OISIVETÉ À L'INSÉCURITÉ : LES INTERSTICES DANS LE CHAMP DE L'ÉDUCATION AU REGARD DE LA MÉTHODE DE LA THÉORIE ENRACINÉE
}

Aude Kerivel

Champ social | « Spécificités »

2015/1 nº 7 | pages 111 à 127

ISSN 2256-7186

ISBN 9782353719068

Article disponible en ligne à l'adresse :

http://www.cairn.info/revue-specificites-2015-1-page-111.htm

\section{Pour citer cet article :}

Aude Kerivel, « Réflexion discursive sur les interstices. De l'oisiveté à l'insécurité : les interstices dans le champ de l'éducation au regard de la Méthode de la théorie enracinée », Spécificités 2015/1 ( $\mathrm{n}^{\circ}$ 7), p. 111-127.

Distribution électronique Cairn.info pour Champ social.

(C) Champ social. Tous droits réservés pour tous pays.

La reproduction ou représentation de cet article, notamment par photocopie, n'est autorisée que dans les limites des conditions générales d'utilisation du site ou, le cas échéant, des conditions générales de la licence souscrite par votre établissement. Toute autre reproduction ou représentation, en tout ou partie, sous quelque forme et de quelque manière que ce soit, est interdite sauf accord préalable et écrit de l'éditeur, en dehors des cas prévus par la législation en vigueur en France. Il est précisé que son stockage dans une base de données est également interdit. 


\title{
Réflexion discursive sur les interstices
}

\section{De l'oisiveté à l'insécurité : les interstices dans le champ de l'éducation au regard de la Méthode de la théorie enracinée}

\author{
Aude Kerivel \\ Docteure en Sociologie, LERFAS, Tours \\ aude.kerivel@hotmail.fr
}

Auteure d'une thèse de doctorat en sociologie intitulée « Jeunesses et respect dans une société fragmentée » soutenu à Paris Descartes, sous la direction de Jan Spurk. Elle est actuellement chercheur au Laboratoire d'Études et Recherche en Action Sociale (LERFAS), elle travaille sur les jeunesses, les trajectoires, les questions de violence et de harcèlement. Ainsi que les organisations, les professionnels, les usagers du champ de l'action sociale et les politiques publiques.

\section{Résumé}

« Non-lieu », « entre-deux », zone d'incertitude, d'anomie ou encore de décharge émotionnelle, telles sont les notions explorées dans un va et vient entre terrain et théorie. A partir d'observations de bagarres entre adolescents dans les temps libres des centres de vacances, la méthodologie de la théorie enracinée nous conduit à interroger le concept d'interstices dans nos sociétés contemporaines. C'est l'envers du décor que nous présentons ici : le cheminement de la recherche fait d'observations, d'hypothèses tantôt réfutées, tantôt confirmées, d'expérimentations, d'intuition et de montée en théorisation.

Mots Clefs : Interstice, Théorie Enracinée, Violence, Liberté, Insécurité 


\section{Introduction}

Nommés friche et non-lieu par les urbanistes qui s'interrogent sur l'espace, zone d'incertitude par les sociologues pour désigner ces temps qui échappent aux mailles de l'organisation, les mots pour parler des «entre-deux» sont souvent imprégnés d'une subjectivité très marquée. Le point de départ de cette réflexion l'est tout autant, puisqu'il interroge «les temps libres » dans le champ de l'animation, du point de vue du professionnel dont la fonction est «d'animer » des groupes d'adolescents. À partir de ce premier objet qui conserve un caractère provisoire et qui sera modifié tout au long de la recherche, nous tenterons de mettre en lumière un cheminement pour une théorisation de la notion d'interstice. Pour arriver à cet objectif, nous utiliserons le cadre méthodologique de la théorie enracinée qui consiste à «produire de la théorie dans un va-et-vient entre proximité et distance, accès à l'information et production d'hypothèses, observation et interprétation des faits » (Glaser et Strauss, 2010). L’idée est de présenter des premiers résultats, mais surtout une démarche méthodologique particulière. En effet, passant tour à tour de la posture de professionnel de terrain à celui de sociologue, l'observateur est plus que participant pour les besoins de l'enquête, il est en immersion. Nous pouvons donc parler d'approche inductive, «par laquelle l'immersion dans les données empiriques sert de point de départ au développement d'une théorie sur un phénomène et par laquelle le chercheur conserve toujours le lien d'évidence avec les recherches de terrain »(Guillemette, 2006, p. 33). Le recueil de données ne se fait pas uniquement au début de l'enquête. La proximité avec le terrain permet d'y retourner tout au long de la recherche afin de vérifier chaque nouvelle hypothèse théorique, mais aussi de faire émerger des hypothèses empiriques « au risque » que celles-ci viennent remettre en cause les résultats de la recherche.

L'un des axes de la procédure méthodologique que nous tenterons de mettre en relief ici, c'est l'interaction circulaire itérative entre la collecte et l'analyse des données. Nous faisons le choix de présenter l'élaboration discursive d'une théorie de l'interstice :

«La formulation d'une théorie sous forme discursive donne une impression de “'développement perpétuel”' qui lui donne toute sa richesse, sa densité et son caractère englobant et rend sa concordance et sa pertinence faciles à comprendre » (Glaser et Strauss, 2010) 
Nous tenterons donc de mettre en lumière, au travers de l'interrogation qui est la nôtre, l'idée selon laquelle «l'adéquation d'une théorie ne peut pas être séparée du processus par lequel elle est produite » (Glaser et Strauss, 2010) D'où la nécessité d'évoquer les va-et-vient entre terrains et théories, les erreurs et les tâtonnements, mais aussi le parcours du chercheur. En effet, la théorie se construit à partir des données de l'enquête, mais également d'autres enquêtes et parfois d'éléments biographiques de l'auteur. Les «intuitions soudaines enracinées en dehors des données » (Glaser et Strauss, 2010) rarement explicitées dans la présentation de la méthodologie, sont déterminantes pour comprendre les résultats et donc les faire évoluer par le repérage de variables ou de nouvelles hypothèses.

Le parcours du chercheur, et les statuts et fonctions occupés par celui-ci sont nécessaires à considérer. Ici, les rôles d'animatrice, de directrice de séjour, de formatrice, d'étudiante en sociologie, de sociologue diplômée, mais aussi de citoyenne ainsi que les rencontres théoriques, et plus globalement ce qui fait «événement», deviennent des données de contexte permettant de comprendre le raisonnement. Dans cet article, nous utiliserons la première personne du singulier lorsque nous parlons en tant qu'acteur et la première personne du pluriel, par convention, lorsque nous nous situons du point de vue de la recherche. Mais le «je » de l'action et le « nous » réflexif vont parfois se confondre.

Nous nous intéressons ici à la manière particulière dont se construit une théorie à partir d'un «problème » de terrain : à savoir la violence dans le champ de l'animation. Les processus de montée en théorie sont rarement visibles dans leur totalité dans les articles et ouvrages scientifiques. Le cadre méthodologique ne s'attarde pas réellement sur les détours et ce qui se produit en dehors de l'outil prévu. C'est donc au cheminement de la réflexion auquel nous nous intéressons ici, de manière chronologique, ou comment théorie et action peuvent être intimement mêlées, s'auto-nourrir et se développer dans un va-et-vient. Cette progression va nous amener progressivement à déplacer notre objet et reformuler une problématique centrée sur la question de l'interstice.

«Si enquêter, c'est identifier des données et les articuler afin de construire une compréhension du problème permettant de progresser de manière nécessaire vers la solution i.e. vers la formulation d'une hypothèse explicative - comme le propose notamment la théorie ancrée, alors il reste à expliciter le moteur de cette progression »(Glaser et Strauss, 2010).

Après avoir présenté le premier problème à l'origine de la réflexion, à savoir la question de la violence pendant les temps libres dans le champ de l'animation, nous expliciterons notre recours à des théories formelles. Puis, bousculant les théories, le terrain va 
faire émerger de nouvelles hypothèses. Celles-ci éclairées par différents courants sociologiques et l'ouverture sur d'autres disciplines vont permettre l'élargissement du concept. Enfin de nouvelles expérimentations sur le terrain vont progressivement faire cheminer la démarche. Les comparaisons avec des terrains comparables et non comparables vont nous amener à une généralisation d'une théorie de l'interstice.

\section{Violence et danger dans les temps libres du quotidien}

Le point de départ est un problème perçu du point de vue d'un acteur de terrain dans son contexte professionnel. Je suis jeune animatrice en centre de vacances et de loisirs (colonie de vacances), n'ayant aucune connaissance en sociologie. Nous pouvons donc prendre au pied de la lettre l'idée selon laquelle le chercheur devrait au moment de l'observation procéder à une «suspension temporaire du recours à des cadres théoriques existants » (Glaser et Strauss, 2010). C'est avec ce statut et les fonctions qui y sont liées que j'effectue ces premières observations. Je constate donc - et mes collègues semblent être d'accord avec ce constat - un certain nombre de bagarres plus ou moins importantes et d'accidents pendant les temps libres. Avec le recul, deux exemples qui me semblent les plus anciens et les plus marquants me reviennent. Le séjour est organisé par l'association «des services sociaux ", c'est un camp d'adolescents dans les Hautes-Alpes. Nous sommes en 1997. Avec le recul, je préciserai que les jeunes accueillis sont issus de classes populaires de milieu urbain, périurbain et rural. Ayant moi-même été accueillie en tant qu'adolescente dans ces séjours, je n'ai pas tout de suite conscience que la population est perçue comme «plutôt difficile ».

La première situation est la suivante : juste après le repas du soir, lors d'un «temps libre », deux jeunes ont des échanges qui rapidement tournent aux insultes et aux menaces. L'un a traité l'autre de «fils de pute », ce dernier, ayant perdu sa mère, a réagi face à ce propos. Le temps d'arriver jusqu'à la scène qui se passe au-dessus de l'emplacement du feu de camp où j'organise la veillée avec un groupe de jeunes, le premier semble s'apprêter à cogner la tête du deuxième avec une grosse pierre.

L'autre situation se passe durant le «temps libre de l'après-midi ». Passant outre l'interdiction de plonger d'un côté de la rivière «pas assez profond » et «semblant présenter quelques dangers », un adolescent «ne remonte pas à la surface » : c'est le propos que nous tiennent les jeunes qui l'accompagnaient lorsqu'ils nous alertent. Ayant la fonction de 
«surveillant de baignade » je saute et remonte le jeune à la surface. Celui-ci respire et peut parler, mais ne peut plus bouger. Nous pensons donc à la colonne vertébrale et appelons aussitôt les pompiers.

Ces situations ont «fait événement» dans mon expérience professionnelle. Nous pouvons d'ailleurs noter que ma posture d'acteur, mon statut de jeune animatrice débutante, ainsi que l'émotion, voire la prise de risque qu'ont suscité ces évènements peuvent expliquer qu'ils existent encore dans ma mémoire. Halbwachs, dans son ouvrage: La mémoire collective (Halbwachs, 1997), observe que les souvenirs sont liés à ce qui « fait événement » pour l'individu. Ainsi en comparant les souvenirs d'individus ayant vécu la même situation, il s'aperçoit que les souvenirs diffèrent. Halbwachs prend notamment l'exemple du professeur qui enseigne. Ce qu'il raconte, la structuration de son cours, mais aussi les exemples et les anecdotes sont de l'ordre d'une technique professionnelle routinière alors qu'ils peuvent faire évènement pour les étudiants qui l'écoutent. Ces situations de violence, si elles sont marquantes, «font exception » et sont loin d'être représentatives de ce qu'est le quotidien des temps libres en colonie de vacances. Pourtant, ce sont celles qui viennent à l'esprit de l'animatrice que je suis à ce moment-là. Lorsqu'on interroge les jeunes sur leurs souvenirs de ce même séjour, ceux-ci sont différents. S'ils se souviennent de la dernière situation, ils évoquent d'abord les relations amicales et amoureuses qui se nouent dans ces temps de la vie quotidienne. Pour eux, la violence n'est pas associée à celle de temps libre.

La question qui se pose est la suivante : «Pourquoi et comment l'observateur voit-il ce qu'il voit? Quelle intuition (au sens originel de regard attentif) a-t-il eue pour concentrer son attention sur ce fait ?» (Glaser et Strauss, 2010)

Les bagarres et accidents qui ont lieu pendant les temps libres sont des accidents à résoudre, pour l'animateur qui observe de son statut de professionnel et pour qui la mission est de veiller à la sécurité des jeunes qu'il accueille. Au-delà de la sécurité des jeunes euxmêmes, c'est la sécurité du statut de l'animateur qui est en jeu.

\section{Le concept d'anomie comme éclairage sociologique}

Face à cette situation de terrain, l'étudiante en sociologie que je suis va utiliser une théorie formelle, celle d'Emile Durkheim, souvent étudiée dans les premières années d'études universitaires. Le temps libre devient un temps entre deux règles, entre deux temps, un temps 
«d'anomie». Nous entendons par anomie «une situation dans laquelle les règles qui permettent à l'ensemble des individus de vivre en harmonie au sein de la société disparaissent ou deviennent inefficaces pour organiser leurs rapports » (Durkheim, 1893). Nous arrivons à la conclusion suivante : les temps libres sont des temps où les règles, les normes, les lois ne sont plus présentes. Ce que nous interprétons comme une absence de «régulation» conduirait donc à des déviances aux normes et nous englobons les bagarres et accidents sous le concept de violence. Nous nous servons dans un premier temps d'une théorie formelle que nous appliquons à un domaine substantif, «une manière classique d'utiliser une théorie logico déductive » (Glaser et Strauss, 2010). Mais si ce procédé amène à l'élaboration d'hypothèses, nous voyons ici que nous nous forçons à « concorder » avec la théorie d'Emile Durkheim sur l'anomie en repérant dans les temps libres tous les évènements « anomiques », du bruit à la désorganisation, en passant par les situations de violence.

La pédagogie, dans laquelle je baigne sans m'en rendre compte en tant qu'acteur, à savoir «l'éducation nouvelle » à la base de projet éducatif tel que celui des Ceméa (Centre d'Entrainement aux Méthodes d'Éducation Actives), m'amène à l'analyse suivante : il faut avoir fait siennes les règles pour pouvoir se les imposer dans un interstice, et ainsi, être «libre et autonome ». En d'autres termes, la mission de l'animateur serait de faire en sorte que les jeunes intériorisent les règles et qu'ils «les emportent avec eux » en permanence même lorsque nous ne sommes, en tant qu'animateur, pas là pour leur rappeler.

En 2001, je deviens directrice du séjour organisé par les services sociaux. J'effectue ce que nous pouvons appeler une première tentative pédagogique. Je restreins très fortement les temps libres afin de réduire le nombre de situations de violence. Les résultats de l'expérience sont visibles et reconnus par tous : l'équipe d'animation (qui peut comparer avec les séjours précédents), le directeur de l'association et même les jeunes. La satisfaction de l'ensemble des acteurs m'amène à la conclusion suivante : en retirant les zones d'anomie, entre deux systèmes de règles, de normes, nous évitons ainsi les situations de violence qu'elles provoquent.

A posteriori, nous pouvons nous demander si la satisfaction de l'ensemble des acteurs vient de cette expérimentation ou du sentiment de sécurité dans lequel se retrouvent les professionnels grâce à cette organisation qui réduit les zones d'incertitudes.

De plus, si nous nous décentrons du présent, et envisageons l'intérêt pédagogique de cette action sur le long terme, cette situation n'est-elle pas quelque peu éloignée de la réalité 
de la vie à laquelle nous préparons les jeunes ? Cette question, je ne me la pose pas à ce moment-là. Mon problème majeur, en tant que directrice de séjour, étant résolu.

Et si le concept d'anomie ne suffisait pas à comprendre les temps libres ? Quand le terrain remet en cause la théorie

Notons ici que jusqu'à présent, la théorie n'avait pas été remise en cause. En effet, ayant à partir de 2001 toujours occupé la fonction de directrice de séjour, ou de responsable d'équipe d'animation en centre sportif et maison de quartier, je n'ai jusqu'à l'expérience présentée ci-après pas eu l'occasion de remettre en cause la théorie formelle utilisée et devenant explication d'un choix pédagogique. Mais cette première explication théorique et sa mise en œuvre dans la pratique ne vont pas résister au terrain.

En 2003, j'ai l'occasion de partir en tant qu'animatrice avec un directeur expérimenté à Cuba. Les jeunes sont issus d'un comité d'entreprise ayant l'habitude de partir ensemble, et venant de milieux socio-culturels variés (tous les échelons d'une entreprise). Les temps libres, nombreux de mon point de vue, se passent «tranquillement» et de nombreux échanges informels ont lieu. Les jeunes et l'équipe d'animation sont satisfaits.

En tant qu'animatrice, je me sens « inutile » de n'avoir aucune situation « de bagarre » à gérer. Avec le recul, je peux observer que j'évolue dans un milieu professionnel où la gestion des «cas difficiles» est une compétence reconnue par le groupe de pairs professionnel. Nous pouvons comparer aujourd'hui ce phénomène avec le « capital guerrier » dont parle Thomas Sauvadet (2006) pour appréhender les « relations entre jeunes des cités ». Selon lui être «un chaud» ou «venir d'un quartier chaud» permet dans certains contextes « d'être respecté ». Entre certains animateurs, nous pouvons observer que le fait d'avoir une expérience avec des jeunes considérés comme «difficiles» ou «chauds » permet d'être « respecté » et reconnu en tant qu'animateur. Suite à ce séjour, dans mes souvenirs, je suis étonnée de l'attachement des jeunes vis-à-vis de moi, j'ai eu le sentiment de ne pas beaucoup agir, de ne pas être «très utile », d'autant plus que le nombre d'animations est contraint par un emploi du temps de visites, dont j'ai peu l'habitude. Il semble s'être passé des choses pendant ces «temps libres », mais je n'en ai pas réellement conscience.

De retour dans des fonctions de directrice de séjour plus habituelles, et consciente que pour se construire, les jeunes ont besoin de tester l'intériorisation des normes et des règles pendant les temps libres, je tente une nouvelle organisation. 
Les séjours, que j'ai l'habitude de diriger sont organisés par le conseil général des Bouches-du-Rhône. Les jeunes participants sont issus de familles d'origine populaire. Ils vivent dans les quartiers nord de Marseille, quartier populaire repéré comme Zone Urbaine Sensible par les politiques de la ville et de ce fait souvent stigmatisé.

Suite à l'expérience précédente, et afin de permettre aux jeunes d'accéder « à une forme d'autonomie », je propose chaque semaine une demi-journée de «temps libres » en faisant en sorte que l'équipe d'animation soit vigilante aux éventuelles violences. Je les alerte en effet sur le fait que ces moments sont propices à ces situations.

La réaction des jeunes, marquante, face à cette « expérimentation » (qui est nommée comme telle a posteriori), est la suivante : les jeunes disent «s'ennuyer » et sollicitent constamment l'équipe d'animation. L'équipe d'animation se serait-elle rendue « indispensable » et aurait-elle été à l'origine de la construction d'un rapport de dépendance ? Cette question, je ne me la pose pas à ce moment-là. Me revient alors un propos souvent entendu dans le milieu de l'animation et par les animateurs eux-mêmes : «les jeunes ne savent plus s'occuper seuls ».

Les animateurs, en allant chercher les causes d'un problème comme le seul fait d'une évolution de la population accueillie empêchent de considérer l'ensemble des facteurs et notamment l'impact de l'évolution d'une organisation. Par exemple, avec la professionnalisation de l'animation et la concurrence des organismes proposant des séjours de vacances, ceux-ci se sont pourvus en activités diverses et variées laissant moins de temps inoccupés.

Face à la réaction des jeunes, émergent de nouvelles hypothèses qui m'obligent à réinterroger terrain et théorie. Je me questionne particulièrement sur ces temps libres et sur ce que je perçois. Je suis consciente que les jeunes ont besoin de tester les normes intériorisées préalablement dans ces espaces, d'expérimenter leur capacité à entrer en relation sans la médiation d'un animateur, mais la satisfaction des jeunes, des animateurs ainsi que la mienne en tant que directrice lorsque je supprime les temps libres me semble être une contradiction que je n'arrive pas à résoudre. Pour nourrir ma réflexion, je vais faire appel à un certain nombre de théories empruntées à la sociologie, mais aussi à d'autres disciplines. 
Lorsque le chercheur arrive sur un terrain, sa trajectoire, ses dernières lectures, ses compétences et son identité de chercheur font qu'il a toutes les chances d'arriver avec une, voire plusieurs théories. Le professionnel de terrain, voire l'usager, a un « problème », mais n'a pas de théorie. D'où l'intérêt de partir de son propos, de ses interrogations. Dans le cas précis, au moment de la première observation, le chercheur n'est pas encore chercheur, il est simple acteur de terrain. Il a des enjeux d'acteur de terrain et une vision très partielle de la situation dans laquelle il se trouve.

Cette posture n'est pas commune, peut-être le chercheur la retrouve-t-il par moment, lorsqu'il est lui-même surpris, voire touché par un événement, une situation qu'il ne comprend pas, ou lorsqu'il n'a aucune théorie qui lui permette d'expliquer le phénomène. Alors il se retrouve dans cette démarche proposée par Glaser et Strauss : «des données, puis la théorie ». Dans le cas présent, ce sont des situations concrètes qui posent problème et interpellent un acteur. Il y a donc des données, fruits d'observation sur le terrain.

Dans le cas présenté ici, c'est le retour au terrain, et plus précisément des observations sur un nouveau terrain qui permettent de « rester fidèle à son corpus de données » (Glaser et Strauss, 2010) et peut-être se permettre des infidélités à la théorie, peut-être même à la discipline.

\section{L'interstice : un espace-temps de désorganisation sociale et de décharge}

\section{émotionnelle}

A ce moment en licence de sociologie, j'étudie Marx, Bourdieu, Passeron. La théorie des classes sociales (Marx, 1867 ; Bourdieu, Passeron, 1964 ; Bourdieu, Passeron, 1970), et des rapports de dominations me marque particulièrement. Nous faisons alors une première hypothèse. Les jeunes qui ne savent pas s'occuper pendant les temps libres sont les jeunes de milieu populaire, que je compare aux «classes dangereuses» en permanence «sous contrôle ». N'ayant jamais l'occasion d'expérimenter des temps libres, ils s'y sentent en insécurité.

La sociologie urbaine nous permet de faire un parallèle qui conduit à élargir la notion de temps libre à partir de l'étude d'une population comparable. L'entre-deux peut s'observer aussi du point de vue de l'espace. L'étude du travail de Trasher (1927) m'amène à une nouvelle hypothèse. En effet, sur son terrain d'enquête que sont les «quartiers pauvres » de 
Chicago, il observe que la rue est «un espace interstitiel » propice à l'émergence de gang. Selon lui, le gang est une réponse à une désorganisation sociale.

La description qu'il fait des membres des gangs est la suivante : «garçon recelant une énergie inemployée qu'aucun modèle social désirable n'est capable de contrôler » (Trasher, 1927).

A partir de ce moment, nous parlons d'interstice, et considérons l'entre-deux temps, mais aussi l'entre-deux espaces. Désormais, ma volonté est de ne pas entrer dans une logique de contrôle des jeunes « des classes dominées »; je souhaite leur laisser les mêmes marges de manœuvre que les jeunes «des classes dominantes». Aussi, je tente de trouver des «arguments pédagogiques » me permettant de gérer au mieux ces interstices, en partant du principe selon lequel les interstices sont des espaces-temps particuliers entre deux «activités » ou deux «endroits » dans lesquels les jeunes n'ont plus de repères.

Pour vivre ces changements, les jeunes ont besoin de repères, tel que le soutient Winnicott (1975). En m'appropriant la théorie des types de jeu de ce même auteur, je propose différentes postures de l'animateur qui jouent le rôle de repère que je présente dans mon projet pédagogique. Dans les différentes situations éducatives, les animateurs sont dans les postures de « laisser jouer », « donner à jouer », «faire jouer », et « jouer avec ».

«En temps calme, après le repas du midi ou avant le repas du soir, on est en laisser jouer. L'animateur est présent, occupé dans un coin, ou discute de manière informelle avec les jeunes. Lorsqu'on laisse un quartier libre aux jeunes en ville on est en donner à jouer, il y a des règles : un horaire à respecter. Pour les activités on est en faire jouer ou jouer avec parfois il peut y avoir un animateur arbitre qui fait jouer et un animateur dans chaque équipe en jouer avec. » (extrait projet pédagogique LPM, Baratier, Juillet 2002).

La conceptualisation a permis de se décentrer de la question du temps «libre ». En imaginant un rôle, une mission à remplir par le professionnel dans ces interstices. Ainsi, nous voyons ici que l'action est dirigée vers l'équipe d'animation et non vers le jeune. La pensée structuraliste a peut être influencé un regard qui se porte davantage sur la structure.

Mais revenons à la réflexion théorique. Une dernière approche va entraîner un changement important dans l'appréhension du phénomène. En cherchant des éclairages théoriques du concept nouvellement découvert d'interstice, nous découvrons le psychanalyste 
Roussillon (Roussillon, 1995) qui présente les interstices comme des espaces de « décharge émotionnelle ». L'appropriation sociologique entraîne l'explication suivante : nous jouons des rôles en fonction des statuts que nous occupons dans les différents groupes auxquels nous appartenons, et ces rôles nous imposent parfois une retenue, un effort. Les interstices sont donc des zones de décompression «nécessaire », avant de ré-endosser un nouveau rôle. C'est ce qui explique la tension dans les transports en commun par exemple. En réinterrogeant l'objet de départ, à savoir les temps libres, je me souviens qu'au-delà de la violence et des accidents, il y a aussi des pleurs et des confidences. Mais ces évènements « me posant moins de problèmes en tant que directrice de séjour » ne m'étaient pas apparus jusqu'à présent. La théorie permet ici de réinterroger des données de terrain qui n'avaient pas été repérées. Suite à cette nouvelle découverte, un retour sur le terrain est nécessaire.

\section{Expérimentation d'organisation pour une gestion des temps libres}

A nouveau directrice de centre de vacances, j'expérimente mes nouvelles réflexions. Parmi les expériences menées lors de différents séjours, je présenterai un exemple d'organisation de «temps libres » et un exemple de gestion d' « un espace interstitiel ». Sans être tout à fait consciente à ce moment que ces dispositifs ont autant pour objectif de répondre aux besoins de sécurité des jeunes qu'à ceux des professionnels.

Lors d'un séjour d'été en Espagne, avec cinquante jeunes entre 15 et 17 ans, en prenant en compte les besoins et demandes de temps libres de certains et d'activités des autres, je propose trois soirs par semaine un «temps libre en ville » aux jeunes, ou la possibilité de faire une sorte de «rallye » dans la ville (avec des petites épreuves, ou des informations à recueillir grâce à un petit carnet construit par l'équipe d'animation). Nous observons que certains jeunes choisissent de profiter du temps libre, d'autres s'emparent du jeu, d'autres font quelques épreuves et profitent également du temps libre, s'autorisant à ne faire le jeu qu'à moitié. Les règles de délimitation du temps et de l'espace de l'interstice (horaires de début et de fin du temps libre, et la zone dans lequel il se passe) semblent permettre aux professionnels d'être en sécurité. Lors d'un séjour d'hiver dans les Hautes-Alpes avec une quarantaine de jeunes entre 12 et 17 ans, je veille à ce que les interstices que constituent les escaliers et les couloirs ne soient pas des zones «d'anomie ». À tour de rôle et lorsque cela est possible, nous (l'équipe d'animation) préparons les jeux ou les veillées, faisons la comptabilité, écoutons de la musique et lisons le journal dans le couloir ou sur les marches d'escalier. Ainsi ces lieux deviennent des interstices occupés. Nous pouvons également noter 
que les professionnels peuvent, en occupant cette place, effectuer une forme de contrôle permanent sur les allers et venues des jeunes. Cette organisation donne satisfaction à l'ensemble des acteurs (jeunes et professionnels), les couloirs et escaliers sont particulièrement investis, et s'improvisent régulièrement des «boums de couloirs ». Cette observation amène plusieurs interrogations : l'interstice est-il toujours interstice lorsqu'il est occupé ? Pourquoi en vient-on à remplir ce vide ? Qui est en insécurité dans ces moments et ces espaces? Les jeunes ou les professionnels? Il semble que malgré tout, la théorie formelle semble avec le recul influencer considérablement les actions de terrain qui consistent à tenter de réduire l'anomie.

\section{Salle d'isolement, couloirs, transports, temps de pause, les différentes formes d'interstice}

L'utilisation de l'analyse comparative va permettre de poser les balises pour une montée en théorisation. En effet, «l'analyse comparative : support pour produire de la théorie, lui redonne sa pleine généralité en l'appliquant à des unités sociales de n'importe quelle taille, grande ou petite, allant des individus ou de leurs rôles sociaux jusqu'aux nations ou aux grandes régions du monde » (Glaser et Strauss, 2010). Les premières comparaisons présentées se sont faites entre des populations présentant un nombre important de similitudes, tentant de tendre vers le précepte «toutes choses égales par ailleurs ». Ce sont des groupes de jeunes en centre de vacances. Le cadre organisationnel est approximativement le même. Les lieux et parfois l'équipe de professionnels, voire une proportion importante de l'équipe de jeunes est la même. Nous arrivons ici à une forme de saturation des données. De plus, en changeant de terrain, le regard «neuf» permettra sans doute d'effectuer de nouvelles hypothèses.

La fonction de sociologue va nous permettre d'effectuer des comparaisons avec d'autres populations qui n'ont comme caractéristiques que le fait d'être jeunes et d'appartenir au champ de l'éducation : Institution scolaire, puis Institution spécialisée (Maison d'enfants à caractère social, Foyer de jeunes) puis Institut thérapeutique éducatif et pédagogique. La comparaison entre groupes permet d'attirer notre attention sur les similitudes et les différences. C'est aussi le passage d'une posture d'observateur participant à celle d'observateur désengagé et le changement de statut du chercheur entraînant une distanciation progressive de l'objet. Parallèlement la définition plus claire et objective du concept d'interstice permet de l'étendre à des terrains plus éloignés de celui de départ. Différents 
exemples permettent d'éclairer encore davantage ce concept. Comme l'avaient perçu les ethnologues, l'étude de l'Autre lointain est souvent plus facile.

Dans le cadre d'une recherche-action, j'accompagne en tant que sociologue des professionnels d'institut thérapeutique éducatif et pédagogique qui visitent des institutions de même nature au Québec. Lors de cette visite, les professionnels (éducateurs, enseignants, directeur d'établissement) sont interpellés par les «salles de contention», les «salles d'isolement » aménagées dans ces lieux qui leur apparaissent comme «des cellules ». Les critiques sont vives pour ces professionnels qui voient dans cette organisation de l'espace l'illustration des théories comportementalistes fortement critiquées en France. D'un point de vue sociologique, je perçois ces salles comme des interstices, possibles espaces pour s'isoler, se reposer du groupe, voire même «se décharger émotionnellement » (Roussillon, 1995).

L'analyse indirecte de données récoltées par d'autres observateurs va permettre d'entrer dans un mode de réflexion déductive. Formatrice en école de travailleurs sociaux, j'interroge la notion d'autonomie, objectif principal de nombreux projets éducatifs qui visent à «permettre à l'enfant d'accéder à une forme d'autonomie ». Nous faisons l'hypothèse que l'organisation du temps dans le champ de l'éducation spécialisée va parfois à l'encontre de toute autonomie. Nous retiendrons deux situations présentées par des étudiants en formation d'éducateur spécialisé. Dans le contexte d'une maison d'enfants à caractère social (MECS) et suite au constat de situations de violence dans les couloirs, un éducateur spécialisé a été nommé « éducateur de couloir » (le titre éducateur de zone de décharge émotionnelle aurait sans doute été plus valorisant !). Chargé de «surveiller les couloirs », il met en place un certain nombre d'activités pendant ces interstices. De l'extérieur, l'évidence surgit. Il « remplit » et surtout utilise la médiation par l'activité pour entrer en relation avec les jeunes accueillis. Les médiations que sont les activités permettent à ce jeune professionnel en construction identitaire d'endosser son nouveau rôle avec l'assurance nécessaire.

Une autre étudiante travaillant en hôpital de jour présente la situation d'une jeune fille dont la «pathologie » est le besoin d'avoir un emploi du temps complètement rempli, le moindre vide déclenchant une crise. Après s'être interrogés sur les interstices des jeunes et notamment de la jeunesse «sous contrôle », nous nous interrogeons sur les interstices des adultes, et de la population qui n'est pas « sous contrôle ». 
Parallèlement, je m'interroge sur mes propres cours qui prennent majoritairement la forme de cours magistraux. N'ai-je pas l'impression de ne pas vraiment «faire mon travail» si je laisse les étudiants autonomes, lire et étudier des textes en petit groupe ? La question du rôle et de la fonction se pose une nouvelle fois et l'on pourrait presque parler ici de «saturation des données ». La comparaison de groupes «non comparables » permet de « transcender » les descriptions substantives circonstanciées et localisées afin de produire une « théorie formelle générale » (Glaser et Strauss, 2010).

\section{La généralisation d'une théorie de l'interstice}

Le travail de conceptualisation amène à interroger en parallèle les concepts suivants : temps libres et zone d'incertitude. «L'observateur participant redéfinit constamment son étude à mesure qu'il découvre de nouvelles données »(Glaser et Strauss, 2010). Après avoir effectué des comparaisons entre populations comparables, il nous semble nécessaire de s'autoriser à des comparaisons avec des populations «non comparables ». Nous présenterons ici les intuitions qui viennent de comparaisons de données « non comparables ».

Posons la question des représentations que nous pouvons avoir du temps libre, du temps libéré, du temps de loisirs. Par mes origines socio-culturelles, j'ai intériorisé le fait que « lire en tant qu'activité de loisir, non productive est assimilé à ne rien faire ». Lors de mes premières années au laboratoire de recherche, je ne m'autorisais pas à lire au bureau. Je réservais donc cette activité à mes week-ends, mais surtout à mes temps de transport. Ces derniers, étant de 5 heures par jour, y trouvaient donc une forme de légitimité. Cette expérience m'a permis d'interroger la subjectivité du fait de ne rien faire que je me rappelle avoir souvent défendu auprès des maîtres de stage des éducateurs de jeunes enfants que je visitais, et qui interprétaient le fait que les étudiants observent comme un non-investissement de leur part. Je fais le parallèle avec certaines conclusions d'une enquête sur les femmes des quartiers sensibles à la recherche d'un emploi. Des mères de famille parlent du temps où elles éduquent leurs enfants et s'occupent de la maison comme un temps où elles ne font rien en opposition avec le temps de travail salarié. Ces deux exemples interrogent la représentation du fait de «ne rien faire » et l'imprégnation religieuse de l'oisiveté comme « péché », « mère de tous les vices ${ }^{1}$.

1 Goffman note que les Institutions fermées souhaitent abolir toutes formes d'oisiveté : «Les périodes d'activités sont réglées selon un programme strict, en sorte que toute tâche s'enchaîne avec la 
Dans ce passage d'une société enchantée (Weber, 1921) à une société rationnelle, ce n'est plus l'oisiveté qui est combattue, mais «l'insécurité ». Même si le premier concept est encore fortement ancré dans les représentations des individus. D'un point de vue journalistique, les interstices sont ce qui n'entre pas dans les rubriques habituelles, collées aux ministères et catégories ministérielles. La banlieue est considérée comme un interstice : hors rubrique, hors catégorie de pensée. Ne parle-t-on pas, dans une surinterprétation, de zone de non-droit? Le contexte idéologique de stigmatisation de l'oisiveté, puis de lutte contre l'insécurité ne peut pas être déconnecté du questionnement et du raisonnement qui est le nôtre. Pourtant, à partir du moment où le fait « de ne rien faire » est subjectif, ne peut-on pas le percevoir comme une capacité à être seul dont parle Hannah Arendt (Arendt, 1961), comme propice à une forme de réflexivité ? En ce qui concerne l'interstice : est-il objectivable? L'interstice des uns est-il l'interstice des autres ? L'interstice des journalistes ou même des politiques qu'est la banlieue n'est-il pas le lieu de résidence de ces habitants ? Les observations faites hors du champ de l'éducation nous conduisent à une montée en généralité, qui permet de réinterroger ce terrain différemment pour une analyse plus fine et objective.

Après ces détours sur d'autres terrains, revenons à la population jeune dans le champ de l'éducation et prenons l'exemple des enfants « placés en institutions : foyer, maison d'enfants à caractère social » qui sont souvent décrits comme des lieux «particulièrement violents ». Cette population fait l'objet d'un travail de recherche en cours, en tant que sociologue. Forte de la réflexion antérieure, nous n'interrogeons plus les interstices du point de vue de l'organisation et des professionnels, maîtres du fonctionnement, mais du point de vue des jeunes, et pas uniquement du point de vue de ce temps précis qui est passé dans telle ou telle institution, mais du point de vue de l'ensemble de leur quotidien. Les jeunes accueillis en foyer se lèvent le matin, mangent dans un lieu collectif, font leur toilette puis prennent un « taxi collectif » pour se rendre à l'école. Là, ils se retrouvent déjà dans un collectif où ils vont devoir jouer un rôle pour se faire respecter selon des normes qui changent : celles de la classe et celles de la cour de récréation. Puis ils vont reprendre le taxi, faire leurs devoirs au foyer, et dîner. Ces jeunes, plus encore que d'autres, n'ont que très peu d'interstices, et explicite de règlements dont l'application est assurée par une équipe administrative." (E.Goffman, 1961, p. 48) 
subissent sans cesse une injonction à jouer un rôle. Être plus dur que l'autre, afin de ne pas se faire «bouffer»sur la grande scène sociale (Goffman, 1973, pp. 25-39) Sur la scène adolescente (Kerivel, 2009), il s'agit d'être insaisissable pour mettre l'autre dans une zone d'incertitude lorsqu'on est soi-même en insécurité.

La confrontation permanente avec les faits, la comparaison et la réflexion discursive menée permet aujourd'hui la montée en théorie. Les bagarres entre adolescents dans les temps libres des centres de vacances nous ont permis d'interroger la subjectivité, diversité, et la fonction des interstices dans nos sociétés contemporaines. Mais cette réflexion nous a surtout permis d'interroger une démarche de recherche faite de va-et-vient entre terrain et théorie. La nécessité de résoudre un problème du point de vue du terrain et le développement progressif d'une analyse théorique ouverte à toutes intuitions, s'autorisant à comparer les terrains, à réfuter les hypothèses et à faire évoluer la problématique, nous semblent des éléments indispensables à la montée en théorie présentée ici. Enfin cet exemple montre que la rigueur méthodologique passe par la mise à jour des détours du chercheur, et des évènements qui dans «les interstices» de la recherche, émergent et influencent considérablement les résultats et la montée en théorisation.

\section{Bibliographie}

Arendt H. (1961). Condition de l'homme moderne. Paris : Calmann-Levy.

Bourdieu P. \& Passeron J-C. (1964). Les héritiers : les étudiants et la culture. Paris : Les Éditions de Minuit.

Bourdieu P. \& Passeron J-C. (1970). La reproduction: Éléments d'une théorie du système d'enseignement. Paris : Les Éditions de Minuit.

Bronner L. (2010). La loi du ghetto, Enquête dans les banlieues françaises. Paris: Pocket.

Durkheim E. (1930/2007, $1^{\text {ère }}$ édition : 1893). De la division du travail social. Paris : Puf.

Kerivel A. (2009). Jeunesses et respect dans une société fragmentée. Thèse de doctorat sous la direction de Jan Spurk, Université Paris Descartes. 
Glaser Barney G., Strauss Anselm L. (2010). La découverte de la théorie ancrée. Stratégies pour la recherche qualitative, Paris : Armand Colin.

Goffman E. (1961). Asiles Études sur la condition sociale des malades mentaux et autres reclus. Paris : Éditions de Minuit.

Goffman E. (1975). Stigmate : Les usages sociaux des handicaps. Paris : Les Éditions de Minuit.

Guillemette F. (2006). L'approche de la Grounded Theory ; pour innover ?, Recherches Qualitatives, vol. 26(1), pp. 32-50.

Halbwachs M. (1997). La mémoire collective. Paris : Albin Michel.

Marx K. (1867/1999). Le Capital. Paris : Gallimard.

Roussillon R. (1995). Logiques et archéologiques du cadre psychanalytique. Paris: Puf.

Sauvadet T. (2007). Le capital guerrier : concurrence et solidarité entre jeunes de cité. Paris : Armand Colin.

Trasher F.M. (1927). The gang. A study of 1313 gangs in Chicago. Chicago: University of Chicago Press.

Weber M. (1921/1995). Économie et société. Paris : Pocket.

Winnicott D. (1975). Jeu et réalité, l'espace potentiel. Paris : Gallimard. 